CASO CLÍNICO

\title{
Carcinoma del cístico, a propósito de un caso
}

\author{
Cystic carcinoma, a clinical case
}

Edson R. Marcos-Ramírez*, María I. Treviño-Martínez, Alejandra Téllez-Aguilera, José M. Ochoa-Aguilar y Francisco Vásquez-Fernández

Servicio de Cirugía General, Hospital Universitario Dr. José Eleuterio González, Universidad Autónoma de Nuevo León, Monterrey, Nuevo León, México

\section{Resumen}

El carcinoma primario en el conducto cístico es un tumor raro y abarca del $0.1 \%$ al $0.2 \%$ de los carcinomas del tracto gastrointestinal. Presentamos el caso de un varón con pérdida de peso e ictericia, en el que se demuestra por imagen una dilatación de vías intrahepática y extrahepática condicionada por una compresión extrínseca del colédoco. Se realiza colangiografía pancreática retrógrada endoscópica que confirma la compresión, por lo que se lleva a cabo una exploración quirúrgica que evidencia una tumoración del conducto cístico. Se realiza colecistectomía con resección de ganglios linfáticos, además de anastomosis hepatoyeyunal. La tumoración se reporta como carcinoma del conducto cístico sin involucro ganglionar.

Palabras clave: Cístico. Carcinoma. Conducto. Ictericia.

\begin{abstract}
Primary cystic duct carcinoma is a rare tumor and comprises $0.1 \%$ to $0.2 \%$ of carcinomas of the gastrointestinal tract. We present a case of a male with weight loss and jaundice. Evidence is shown by imaging of intrahepatic and extrahepatic duct dilatation conditioned by extrinsic bile duct compression. An endoscopic retrograde pancreatic cholangiography was performed confirming the compression, so a surgical exploration was preformed showing a tumor of the cystic duct, a cholecystectomy with resection of regional lymph nodes, as well as a hepatic-jejunal anastomosis. The tumor is reported as cystic duct carcinoma without lymph node involvement.
\end{abstract}

Key words: Cystic. Carcinoma. Duct. Jaundice.

\section{Introducción}

La patología neoplásica en los conductos biliares extrahepáticos es poco frecuente dentro de las neoplasias gastrointestinales, abarcando del $2 \%$ al $3.6 \%$ en su incidencia'. El carcinoma primario en el conducto cístico fue descrito por primera vez en 1951 por Farrar ${ }^{2}$ y desde entonces se han reportado 70 casos en la literatura ${ }^{1,3}$. Es un tumor de muy baja frecuencia que corresponde al $0.1 \%$ a $0.2 \%$ de los carcinomas del tracto gastrointestinal. La manera tradicional de su diagnóstico era al cumplir los criterios descritos, los cuales incluyen crecimiento

\section{Correspondencia:}

*Edson R. Marcos-Ramírez

Avda. Francisco I Madero s/n

Col. Mitras Centro

Fecha de recepción: 06-04-2020

C.P. 64460 , Monterrey, N.L., México

E-mail: ermarcos7@gmail.com
Cir Cir. 2021;89(4):542-546

Contents available at PubMed www.cirugiaycirujanos.com ajo la licencia CC BY-NC-ND (http://creativecommons.org/licenses/by-nc-nd/4.0/) 
restringido al conducto cístico, ausencia de un proceso neoplásico en la vesícula biliar, el hígado o el conducto biliar común, y evidencia histológica de carcinoma ${ }^{1-3}$. Sin embargo, estos estrictos criterios creaban problemas en la práctica clínica, por lo que diversos autores presentaron nuevos criterios que englobaban la enfermedad por extensión metastásica. Con este avance se comenzó a analizar su tratamiento, el cual previamente era solo quirúrgico, sin dar posibilidad a evaluar otras terapias adyuvantes como la radioterapia posquirúrgica ${ }^{1,3-5}$. Debido a los pocos casos descritos en la literatura y a la importancia de la evaluación de estos pacientes, se presenta el siguiente caso que tuvimos como experiencia en el Hospital Universitario de la Universidad Autónoma de Nuevo León, México.

\section{Caso clínico}

Varón de 49 años con antecedente de hepatopatía crónica en estudio y litiasis renal recurrente. Inicia su cuadro con pérdida de peso de $5 \mathrm{~kg}$ en los últimos 2 meses acompañada de ataque al estado general, ictericia y prurito generalizado. Al iniciar su abordaje se encuentra en los estudios de laboratorio leucocitosis, alteración de las transaminasas e hiperbilirrubinemia con predominio de bilirrubina directa: leucocitos 16.9 $\mathrm{K} / \mathrm{U}$, aspartato transaminasa $120 \mathrm{UI} / \mathrm{l}$, alanina transaminasa $140 \mathrm{UI} / \mathrm{l}$, fosfatasa alcalina $911 \mathrm{UI} / \mathrm{l}$, bilirrubina total (BT) $12.5 \mathrm{mg} / \mathrm{dl}$, bilirrubina directa $7.3 \mathrm{mg} / \mathrm{dl}$, bilirrubina indirecta $5.2 \mathrm{mg} / \mathrm{dl}$. En la serología viral se detecta positivo para virus de la hepatitis $\mathrm{C}(\mathrm{VHC})$ y negativo para virus de la inmunodeficiencia humana, por lo que se considera que el origen de la hepatopatía del paciente es viral. Se solicita ultrasonido de abdomen superior, que reporta hígado heterogéneo con dilatación de vías biliares intrahepáticas, vesícula biliar con pared de $2 \mathrm{~mm}$, colédoco de $16 \mathrm{~mm}$ visible hasta la cabeza de páncreas, el cual se visualiza normal. Para caracterizar mejor las vías biliares se solicita una resonancia magnética en la cual se observa la vesícula biliar con cambios hidrópicos, además de una obstrucción del tercio medio del colédoco distal y ectasia de la vía biliar proximal a este punto, sin evidencia de litiasis, tumoración en cabeza de páncreas ni proceso neoplásico que condicionara este punto de dilatación del colédoco (Fig. 1).

Debido a estos hallazgos, se realiza al paciente una colangiografía pancreática retrógrada endoscópica (CPRE) que muestra el ámpula, el cual se logra canular; área de estenosis extrínseca en el colédoco sin

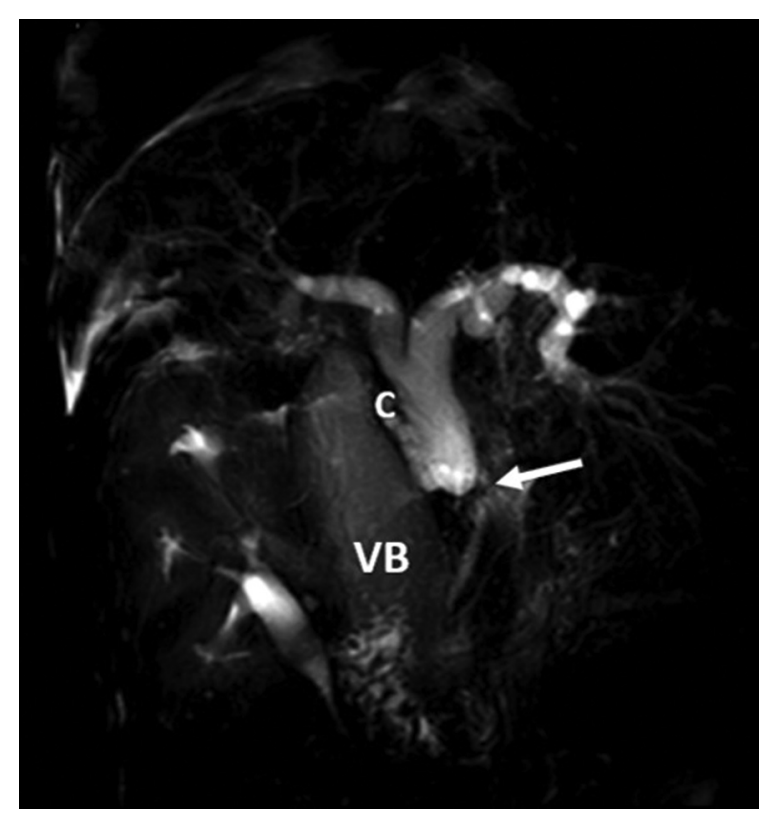

Figura 1. Reconstrucción de resonancia magnética de vías biliares. La flecha indica el punto de transición de la dilatación del conducto colédoco, que coincide con el punto donde el conducto cístico $(C)$ se une al colédoco. VB: vesícula biliar.

evidencia de proceso neoplásico intraluminal; se pasa la cánula, pero no se logra entrar en la vía intrahepática y tampoco pasar al conducto cístico. Se toma muestra para citología y se termina el procedimiento con una esfinterotomía. A pesar de la CPRE, el paciente se mantuvo con hiperbilirrubinemia a expensas de la bilirrubina directa. La citología de líquido biliar reporta material proteínico, biliar y celular sin anormalidades.

Para complementar el estudio se realiza un ultrasonido endoscópico, en el cual se inspecciona la cabeza de páncreas sin hallazgos relevantes y se observa el colédoco extrapancreático marcadamente dilatado (12 mm), con escaso lodo en su interior, sin localización franca de alguna tumoración, y el colédoco intrapancreático de calibre normal ( $3 \mathrm{~mm}$ ). Se observa linfadenopatía regional de aspecto reactivo en la región del hilio hepático (Fig. 2).

Por esos hallazgos se solicita una tomografía computada (TC) de tórax y abdomen con contraste, que reporta dilatación del colédoco de hasta $2 \mathrm{~cm}$ acompañada de dilatación de la vía intrahepática, así como vesícula hidrópica, sin evidencia de lesiones sospechosas de malignidad a distancia en el hígado, los pulmones ni el tejido óseo.

Se decide realizar una exploración quirúrgica, en la que se encuentra el hígado de características 


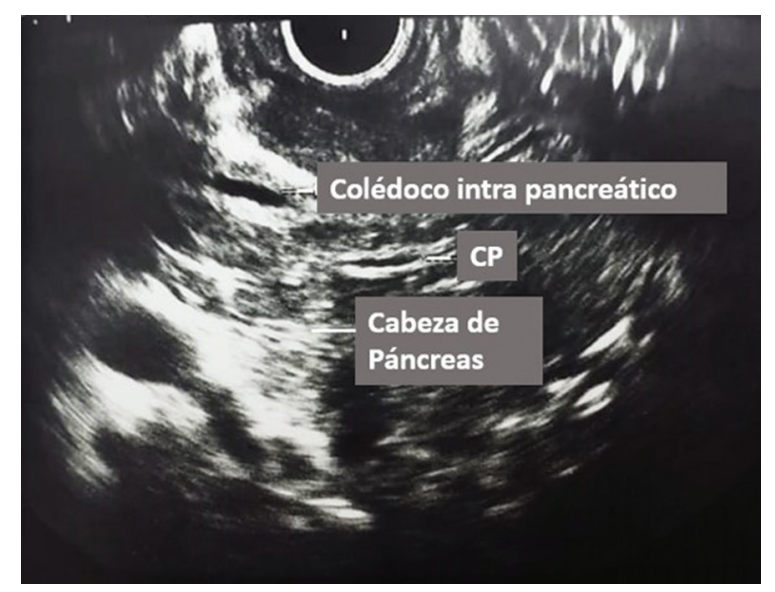

Figura 2. Ultrasonido endoscópico que evidencia la dilatación del conducto colédoco $(12 \mathrm{~mm}$ ) sin proceso neoplásico en la cabeza del páncreas, además del conducto pancreático $(C P)$ normal.

cirróticas generalizadas, se visualiza la vesícula biliar y se identifica el conducto cístico engrosado de tamaño por tumoración propia de la estructura sin infiltración hacia el conducto colédoco, los conductos hepáticos ni las estructuras vasculares en la inspección macroscópica. Se realiza colecistectomía con resección del conducto colédoco en la proximidad a la tumoración del cístico, además de ganglios linfáticos regionales para su estudio histopatológico. Para la reconstrucción de la vía biliar se realiza una anastomosis hepatoyeyunal a $60 \mathrm{~cm}$ del ángulo de Treitz, cursando sin eventualidades el procedimiento quirúrgico. En la figura 3 se muestra la tumoración encontrada en el conducto cístico.

En el posoperatorio inmediato (24 horas), la BT disminuyó a $8.4 \mathrm{mg} / \mathrm{dl}$ con bilirrubina directa de $5.1 \mathrm{mg} / \mathrm{dl}$, y se obtuvieron valores normales de BT $(0.9 \mathrm{mg} / \mathrm{dl})$ a las 72 horas de la cirugía, por lo que se egresó al paciente sin eventualidades.

Se reporta análisis histopatológico de vesícula biliar de $11.6 \times 4 \times 0.6 \mathrm{~cm}$, con datos de colecistitis crónica sin evidencia de células neoplásicas en la vesícula biliar, además de identificar el conducto cístico con dimensiones de $2.5 \times 1 \times 0.9 \mathrm{~cm}$ con un grosor de la pared de $0.4 \mathrm{~cm}$ y una luz de $0.1 \mathrm{~cm}$, observando en el corte histológico células neoplásicas compatibles con carcinoma de conducto cístico en su tercio medio con infiltración a la pared, así como infiltración linfática, perineural y de tejido graso periductal sin infiltración a ganglios linfáticos, y bordes de la resección negativos para malignidad.

Este paciente se catalogó como carcinoma del conducto cístico en etapa clínica IIIA y se mantuvo bajo
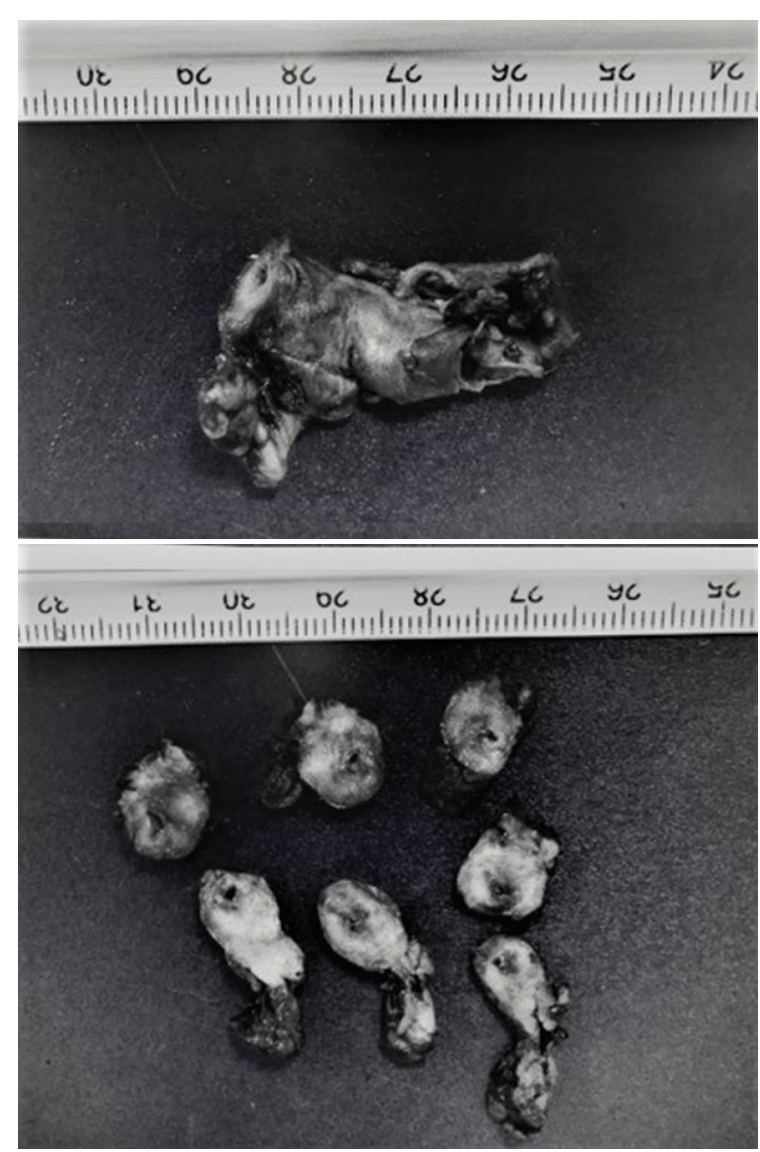

Figura 3. Pieza quirúrgica del conducto cístico seccionado en la que se evidencia el proceso neoplásico. Puede verse el engrosamiento del conducto y la luz de este disminuida de tamaño.

vigilancia oncológica durante los siguientes 12 meses por medio de TC control, sin evidencia de enfermedad residual.

Se realiza una nueva TC durante la vigilancia oncológica al cumplir el segundo año posquirúrgico y se evidencian múltiples adenopatías a nivel del hilio hepático, paraaórtico, aortocava y en el tronco celiaco. Ante la sospecha de una recaída regional se inicia quimioterapia con gemcitabina $\left(1000 \mathrm{mg} / \mathrm{m}^{2}\right)$ y cisplatino (42 mg) cada 21 días; al cuarto ciclo de quimioterapia se cambió la gemcitabina por capecitabina debido a toxicidad.

Actualmente, durante su tercer año posquirúrgico, el paciente se ha mantenido estable, funcional, con enfermedad en primera línea de tratamiento.

\section{Discusión}

El carcinoma primario del cístico tiene una preponderancia masculina y la edad media de presentación 
es de 65 años (rango: 38-79 años) ${ }^{6-9}$. No se ha asociado a enfermedad litiásica vesicular, al encontrarse solo en el $25 \%$ de todos los casos ${ }^{1,8,10,11}$, y tampoco se ha asociado a la presencia de $\mathrm{VHC}^{12}$, por lo que consideramos que en nuestro paciente es una coincidencia y no una asociación etiológica, aunque, a pesar de ser un caso excepcional, estas asociaciones no están descritas en la literatura por la baja frecuencia del padecimiento. Su presentación clínica no es específica, muy similar a la de la litiasis en la vía biliar, pero sus principales síntomas son dolor abdominal e ictericia. En una serie de 33 casos se reportó que el $81 \%$ se presentaban con dolor abdominal en el cuadrante superior derecho, el $41 \%$ asociaban una tumoración abdominal y solo en 4 de los 33 casos hubo ictericia obstructiva ${ }^{6}$. Son muy pocos los pacientes en los que el diagnóstico se realiza preoperatoriamente mediante estudios de imagen ${ }^{2,13-15}$; en la mayoría de los casos, la tumoración se descubre en la laparotomía 0 en el estudio histopatológico del espécimen, como ocurrió en nuestro caso.

Se han desarrollado diversos sistemas para estadificar el carcinoma del conducto cístico, pero no se ha establecido un protocolo diagnóstico aplicable a todos los casos. Los criterios de Farrar son estrictos y no engloban a la enfermedad avanzada, debido a que, según estos, una vez presente la invasión linfática se convierte en un "carcinoma no del conducto cístico»2,4. Por eso, las nuevas clasificaciones diagnósticas definen al carcinoma del conducto cístico como un tumor de localización centrada en el conducto cístico con capacidad de invasión hacia los ganglios linfáticos y las estructuras vecinas, denominación que es más práctica para la mayoría de los casos con estadios avanzados (Tabla 1). La incidencia de metástasis en los ganglios linfáticos es del $40 \%$, la cual se ha reportado baja en comparación con la del cáncer en las vías extrahepáticas $(50 \%)$ o la vesícula biliar $(80 \%)^{7,16,17}$.

El desarrollo temprano de los síntomas debido a la obstrucción del conducto cístico, su crecimiento lento y las metástasis tardías favorecen un mejor pronóstico en los pacientes con carcinoma del cístico 3 3,4,9,16-19. La invasión perineural es uno de los factores pronósticos de riesgo más significativos, con una frecuencia del $85-93 \% \%^{1,11,12,20}$. Nuestro paciente presentaba invasión perineural y angiolinfática.

El tratamiento recomendado es la cirugía radical, que comprende colecistectomía y escisión de los conductos biliares con linfadenectomía regional. La radioterapia adyuvante posoperatoria puede considerarse
Tabla 1. Criterios de Farrar y nuevos criterios diagnósticos del carcinoma del conducto cístico

\begin{tabular}{lc}
\hline Criterios de Farrar & Nuevos criterios \\
\hline - El crecimiento del tumor & - El crecimiento del tumor puede \\
debe estar estrictamente & pasar más allá del conducto \\
restringido al conducto cístico & cístico \\
- No deben existir procesos & - El sitio primario del tumor se define \\
neoplásicos en la vesícula & por el sitio de mayor grosor (centro \\
biliar ni en los conductos & geométrico del tumor), siendo el \\
hepáticos & conducto cístico el sitio anatómico \\
- En el estudio histopatológico, & de este proceso \\
la tumoración debe tener & - Presencia de células de carcinoma \\
presencia de células del & en el estudio histopatológico \\
carcinoma & \\
\hline
\end{tabular}

en los casos de enfermedad avanzada, en particular en aquellos con márgenes quirúrgicos positivos. La supervivencia media es de 27,2 meses, mientras que la del carcinoma de vesícula biliar es de solo 5,8 meses y la de otros conductos biliares extrahepáticos es de 3-11 meses; en nuestro paciente hemos sobrepasado esta supervivencia media, llegando actualmente a los 32 meses de sobrevida.

\section{Conclusiones}

La naturaleza de esta afección se vio sesgada por mucho tiempo debido a unos criterios diagnósticos estrictos, los cuales dejaron fuera de estudio los casos de enfermedad avanzada; sin embargo, con los nuevos criterios diagnósticos se pueden incluir más casos y por consiguiente aumentar la significancia de los estudios prospectivos, haciendo énfasis en la terapia posquirúrgica de la enfermedad avanzada, así como en el seguimiento a largo plazo de los pacientes que presentan enfermedad localizada. En este caso se obtuvo el diagnóstico incidental debido a la mayor frecuencia de casos con colangiocarcinoma que tenemos en nuestro centro; sin embargo, es de gran importancia tener en cuenta esta enfermedad como uno de los posibles diagnósticos diferenciales en los pacientes con ictericia obstructiva y características oncológicas.

\section{Agradecimientos}

Los autores agradecen a cada uno sus pacientes, quienes son una historia nueva que contar y hacen posible el avance del conocimiento. $Y$ al equipo de trabajo y los pacientes de nuestro hospital-escuela, que son la razón de ser de nuestra labor. 


\section{Conflicto de intereses}

Los autores declaran no tner ningun conflicto de intereses.

\section{Responsabilidades éticas}

Protección de personas y animales. Los autores declaran que los procedimientos seguidos se conformaron a las normas éticas del comité de experimentación humana responsable y de acuerdo con la Asociación Médica Mundial y la Declaración de Helsinki.

Confidencialidad de los datos. Los autores declaran que han seguido los protocolos de su centro de trabajo sobre la publicación de datos de pacientes.

Derecho a la privacidad y consentimiento informado. Los autores han obtenido el consentimiento informado de los pacientes y/o sujetos referidos en el artículo. Este documento obra en poder del autor de correspondencia.

\section{Bibliografía}

1. Bains L, Kaur D, Kakar AK, Batish A, Rao S. Primary carcinoma of the cystic duct: a case report and review of classifications. World J Surg Oncol. 2017; 15:30.

2. Farrar DAT. Carcinoma of the cystic duct. Br J Surg. 1951;39:183-5.

3. Kim WC, Lee DH, Ahn SI, Kim JM. A case of cystic duct carcinoma treated with surgery and adjuvant radiotherapy: a proposal for new classification. J Gastrointest Liver Dis. 2007:16:437.
4. Ozden I, Kamiya J, Nagino M, Uesaka K, Oda K, Sano T, et al. Cystic duct carcinoma: a proposal for a new "working definition". Langenbecks Arch Surg. 2003;387:337-42.

5. Yokoyama $\mathrm{Y}$, Nishio H, Ebata T, Abe T, Igami T, Oda K, et al. New classification of cystic duct carcinoma. World J Surg. 2008;32:621-6.

6. Baraka A, Al Mokhtar NY, Madda JP, Amirrad M, Asfar S. Primary carcinoma of the cystic duct causing obstructive jaundice hepatic hydrothorax without ascites. J R Soc Med. 1990;83:746-7.

7. Yamaguchi K, Nishihara K, Tsuneyoshi M. Carcinoma of the cystic duct. J Surg Oncol. 1991;48:282-6.

8. Do JH, Choi YS, Ze EY. Adenocarcinoma developed from remnant cystic duct after cholecystectomy. BMC Gastroenterol. 2014;14:1-4.

9. Felekouras E, Petrou A, Bramis K, Prassas E, Papaconstantinou I, Dimitriou N, et al. Malignant carcinoid tumor of the cystic duct: a rare cause of bile duct obstruction. Hepatobiliary Pancreat Dis Int. 2009;8:640-6.

10. Choi JH, Kim HW, Lee JC, Paik KH, Seong NJ, Yoon CJ, et al. Percutaneous transhepatic versus EUS-guided gallbladder drainage for malignant cystic duct obstruction. Gastrointest Endosc. 2017;85:357-64.

11. Kamisawa T, Kuruma S, Tabata T, Chiba K, Iwasaki S, Koizumi S, et al Pancreaticobiliary maljunction and biliary cancer. J Gastroenterol. 2015;50:273-9.

12. Manabe T. Cystic duct. InnerBody.com. 2015; 2017(April 9). Disponible en: http://www.innerbody.com/anatomy/digestive/cystic-duct.

13. Kawakami H, Kuwatani M, Abe Y, Kubota Y, Kawakubo K, Kubo K, et al. Direct peroral ultraslim endoscopy-guided biliary drainage in a patient with cystic duct carcinoma and an occluded self-expandable metallic stent. Endoscopy. 2015;47 Suppl 1 UCTN: E43-4.

14. Obana T, Fujita N, Noda Y, Kobayashi G, Ito K, Sugawara T, et al. Endoscopic biliary imaging and clinicopathological features of cystic duct cancer. J Gastroenterol. 2008;43:171-8.

15. Shiba $H$, Misawa $T$, Ito $R$, Imazu H, Suzuki M, Yanaga K. Preoperative diagnosis of early cystic duct cancer using endoscopic ultrasonography and endocholangioscopy: report of a case. J Gastrointest Surg. 2011;15:1477-9.

16. Francisco E, Mendes M, Vale S, Esteves J. Cystic duct carcinoma mimicking a middle bile duct tumour. BMJ Case Rep. 2015;2015:bcr2014208813.

17. Chao TC, Greager JA. Carcinoma of the extrahepatic bile ducts. J Surg Oncol. 1991;46:145-50.

18. Modlin IM, Lye KD, Kidd M. A 5-decade analysis of 13,715 carcinoid tumors. Cancer. 2003;97:934-59.

19. Kodaganur S, Hosamani IR. Synchronous malignancies of the gall bladder and common bile duct: a case report. World J Surg Oncol. 2016;14:106.

20. Nakata T, Kobayashi A, Miwa S, Soeda J, Uehara T, Miyagawa S. Clinical and pathological features of primary carcinoma of the cystic duct. J Hepatobiliary Pancreat Surg. 2009;16:75-82. 